\title{
Musculoskeletal Ultrasonography and Magnetic Resonance Imaging in Early Detection of Inflammatory Arthropathy in Patients with Systemic Sclerosis
}

\author{
Lobna AM Habib ${ }^{1}$, Reem A. Habeeb ${ }^{2}$ \\ Departments of Radiology ${ }^{1}$, Internal Medicine ${ }^{2}$, Ain Shams University; Egypt
}

\begin{abstract}
Background: Articular involvement is frequent in Systemic Sclerosis. Joint symptoms have been noted in 24-97\% of patients at some time during the course of their illness. Musculoskeletal Ultrasound and Magnetic Resonance Imaging can identify and characterize subclinical synovial inflammation as well as overt joint damage in Systemic Sclerosis. Aim of Study: To evaluate the role of Musculoskeletal Ultrasound and Magnetic Resonance Imaging in early detection of inflammatory arthropathy in patients with Systemic Sclerosis and to correlate the radiological findings with various disease parameters. Patients and Methods: 20 patients with Systemic Sclerosis and arthralgia, with no evidence of clinical or laboratory features of overlap syndromes (including Rheumatoid Factor or Anti CCP) or evidence of clinically swollen or tender joints, were subjected to clinical and immunological evaluation. Musculoskeletal ultrasonography and Magnetic resonance imaging were done for tenosynovitis, synovitis and erosions, with MRI representing the standard reference method. Results: Musculoskeletal ultrasound detected tenosynovitis in 55\%, synovitis in 50\% and erosions in 35\% of patients as compared to Magnetic resonance which detected tenosynovitis in 80\%, synovitis in $75 \%$ and erosions in 55\% of the patients with a percentage of agreement of $72 \%, 64 \%$ and $60 \%$ for tensosynovitis, synovitis and erosions respectively. Tenosynovitis and synovitis showed a significant positive correlation with ESR ( $\mathrm{r}=0.44)$ and CRP ( $\mathrm{r}=0.53)$ and erosions correlated significantly with the disease duration $(\mathrm{r}=0.63)$. Conclusion: Inflammatory arthropathy and erosive changes are present in a high percentage of systemic sclerosis patients. Tenosynovitis and synovitis correlate with systemic inflammatory markers. Both musculoskeletal ultrasound and magnetic resonance imaging are useful in characterizing early inflammatory arthropathy in systemic sclerosis patients. [Egypt J Rheumatology \& Clinical Immunology, 2015; 3(1): 39-45]
\end{abstract}

Key Words: SSc, arthropathy, MSUS, MRI

\section{INTRODUGTION}

Systemic Sclerosis (SSc) is a connective tissue disease characterized by vascular, immune and fibrotic changes in the skin and internal organs. The majority of patients with SSc develop musculoskeletal symptoms during the course of their illness. Manifestations may include varying degrees of rheumatic complaints ranging from arthralgia to frank arthritis, and with progression of the disease, debilitating contractures may occur. ${ }^{1}$

Joint involvement may be an initial manifestation that precedes the onset of Raynaulds phenomenon or may arise concomitantly and therefore might be considered an early indicator of SSc. The frequency of joint involvement as an initial sign of $\mathrm{SSc}$ has not been defined accurately and ranges from $12 \%$ to $66 \%$ of SSc patients. ${ }^{2}$

Recent cohort studies examining clinical and radiographic aspects of $\mathrm{SSc}$ have clarified the frequencv of articular involvement and identified

For Correspondence: reemhabeeb@yahoo.com subsets of SSc patients with a higher risk of developing joint involvement. They have also highlighted the potential contribution of inflammatory arthritis to early SSc. Some pilot studies have underlined the potential usefulness of Musculoskeletal ultrasonography (MSUS) and Magnetic resonance imaging (MRI) for a better evaluation of joint involvement in $\mathrm{SSc}^{3}$

Furthermore, Erosive joint damage has been reported in SSc patients and has been attributed to overlap with mixed Connective tissue disease or Rheumatoid arthritis. MSUS and MRI can identify and characterize subclinical synovial inflammation as well as overt joint damage in SSc patients with much greater precision than X-ray. ${ }^{4}$

\section{Aim of The Work:}

The aim of this study was to evaluate the role of MSUS and MRI in early detection of inflammatory arthropathy in patients with SSc and to correlate the radiological findings with various disease parameters. 


\section{PATIENTS AND METHODS}

This study was conducted on 20 patients, diagnosed with systemic sclerosis according to the 2013 American Colleague of Rheumatology/European League Against Rheumatism Systemic Sclerosis classification criteria. ${ }^{5}$ Patients were attending the Rheumatology outpatient clinic and the inpatient ward of Internal Medicine department at Ain Shams University Hospital. Informed consents were obtained from the patients and the study was approved by Ain Shams Medical ethics committee.

\section{All patients were subjected to the following:}

Full history taking and thorough clinical and musculoskeletal examination:

All patients were selected with arthralgia with no evidence of clinically swollen or tender joints, and patients with clinical or laboratory features of overlap syndromes (including Rheumatoid Factor or Anti CCP) were excluded from the study.

\section{Laboratory Investigations:}

* Complete blood count and Erythrocyte sedimentation rate (ESR)

* Immunological tests:

- $\quad$ Antinuclear antibody (ANA), Anticentromere antibodies (ACA) and Anti-RNP antibodies by indirect immunofluorescene technique.

- $\quad$ Anti topoisomerase antibodies (AntiScl-70) and Anti-cyclic citrullinated protein antibodies (anti-CCP) with titer using ELISA technique.

- Rheumatoid Factor (RF): measured by using biotic RA factor latex agglutination slide for the qualitative determination of $\mathrm{RF}$ in serum.

\section{Radiological Evaluation:}

\section{Musculoskeletal Ultrasonography:}

Performed using a General Electric LOGIQ 500 unit (General Electric, Solingen, Germany) using a 7$13 \mathrm{MHz}$ linear array transducer. Joints were examined by an expert radiologist with a rheumatological background, blinded to the diagnosis and clinical data. Bilateral $2^{\text {nd }}$ to $5^{\text {th }}$ metacarpo phalangeal (MCP), proximal interphalangeal (PIP), distal interphalangeal (DIP) joints were assessed, as well as the distal radioulnar and radiocarpal joints, inter-carpal and carpometacarpal joints. Tendons (extensor and flexors of the hands and fingers at the level of the wrists and hands) were also examined using a multiplanar and dynamic scanning technique. All explored joints and tendons were evaluated for the presence of synovial hypertrophy on grey scale and synovitis/tenosynovitis on power Doppler ultrasonography, according to
OMERACT definitions criteria. ${ }^{6}$ The following values of Doppler settings were used: frequency 7.5 MHz, low wall filter, pulse repetition frequency ranging from 700 to $1000 \mathrm{~Hz}$ and the maximal gain level not generating artifacts signal below the bony cortex. Bones were examined for erosions, defined as a cortical "break" or defect seen in longitudinal and transverse planes.

\section{Magnetic Resonance Imaging:}

Examination of the wrists and hands was performed using a 1.5 Tesla MR scanner (Achieva; Philips Medical Systems, Bothell, WA, USA). The following conventional pulse sequences were used for all patients: Coronal and axial T1-weighted and T2weighted fast spin echo, coronal short inversion time inversion-recovery (STIR) and contrast-enhanced coronal and axial T1-weighted sequence with fat suppression. The TR/TE values used in T1- and T2weighted imaging were 424/10 and 3000/100, respectively, while STIR images were obtained by using 3761/80 ( TR/TE). Other key imaging parameters acquired were as follows: slice thickness, $3 \mathrm{~mm}$; flip angle $10^{\circ}$; field of view (FOV) $250 \mathrm{~mm}$. MRI images were assessed by a musculoskeletal radiologist (blinded to the diagnosis, clinical data and ultrasound findings) for the presence of synovitis, tenosynovitis, bone marrow edema, and erosions. MRI images were considered the standard reference for confirming the presence of these lesions.

\section{Statistical Analysis}

Statistical analysis was performed using SPSS for Windows version 17.0, two-tailed tests were used. Descriptive statistics of the range, means, and standard deviation were calculated for interval and ordinary variables and frequencies and percentages for categorical variables, correlations were bivariate correlations procedure computes Pearson's correlation and percentage of agreement. For all tests $\mathrm{P}$ value $>$ 0.05 was insignificant and $\mathrm{P}<0.05$ was significant.

\section{RESULTS}

This study was conducted on 20 patients diagnosed as systemic sclerosis. There were 4 males $(20 \%)$ and 16 females $(80 \%)$. Their mean age was 37.3 years (range $32-53$ years) and their mean disease duration was 8.7 years (range 6-19 years).

\section{Ultrasound Findings}

Table (2) shows the ultrasonographic findings in patients. MSUS was able to detect tenosynovitis (Figure $2 \mathrm{~A}$ and $\mathrm{B}$ ) in 11 patients (55\%), and synovitis (Figure $3 \mathrm{~A}$ and $\mathrm{B}$ ) in 10 patients $(50 \%)$. Erosions were found in 7 patients $(35 \%)$ while bone marrow edema was not depicted on US at all (Figure 4C). 


\section{MRI Findings}

Table (3) shows the MRI findings in patients. MRI detected tenosynovitis (Figure 2C) in 16 patients $(80 \%)$, and synovitis (Figure 3C) in 15 patients $(75 \%)$, erosions in 11 patients $(55 \%)$ and bone marrow edema in 13 patients $(65 \%)$ (Figure $4 \mathrm{~A})$.

\section{Comparison between MSUS and MRI}

Table (4) shows the comparison between MSUS and MRI in detecting tenosynovitis, synovitis and erosions. MRI detected tenosynovitis and synovitis in 5 more patients than MSUS (25\%) (Figure 5) and erosions in 4 more patients.

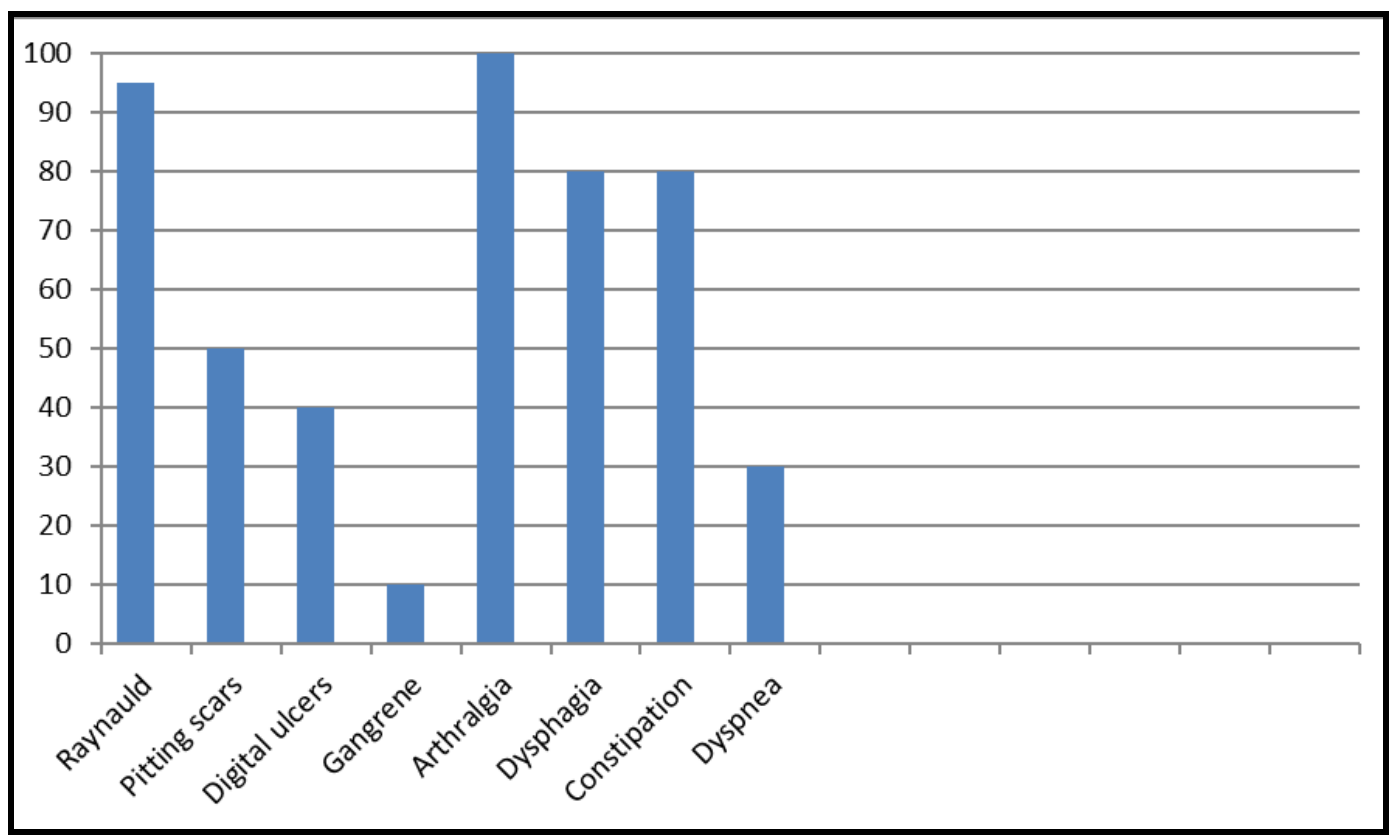

Figure 1. Clinical data of the patients.

Table 1. Immunological data of the patients.

\begin{tabular}{|c|c|c|}
\hline Variable & Number & Percent (\%) \\
\hline CRP (+ve) & 14 & 70 \\
\hline ANA & 19 & 95 \\
\hline Anti-scl-70 & 19 & 95 \\
\hline ACA & 7 & 35 \\
\hline $\mathrm{RF}$ & 0 & 0 \\
\hline Anti CCP & 0 & 0 \\
\hline Anti RNP & 1 & 5 \\
\hline \multicolumn{3}{|c|}{ Mean \pm SD } \\
\hline ESR & \multicolumn{2}{|c|}{$37.3 \pm 24.16$} \\
\hline
\end{tabular}

Table 2. Ultrasonographic findings in patients.

\begin{tabular}{lcc}
\hline \multicolumn{1}{c}{ MSUS Finding } & Number & Percent $(\boldsymbol{\%})$ \\
\hline Tenosynovitis & 11 & 55 \\
Synovitis & 10 & 50 \\
Erosions & 7 & 35 \\
\hline
\end{tabular}


Table 3. MRI findings in patients.

\begin{tabular}{lcc}
\hline \multicolumn{1}{c}{ MRI Finding } & Number & Percent $(\boldsymbol{\%})$ \\
\hline Tenosynovitis & 16 & 80 \\
Synovitis & 15 & 75 \\
Erosions & 11 & 55 \\
Bone marrow edema & 13 & 65 \\
\hline
\end{tabular}

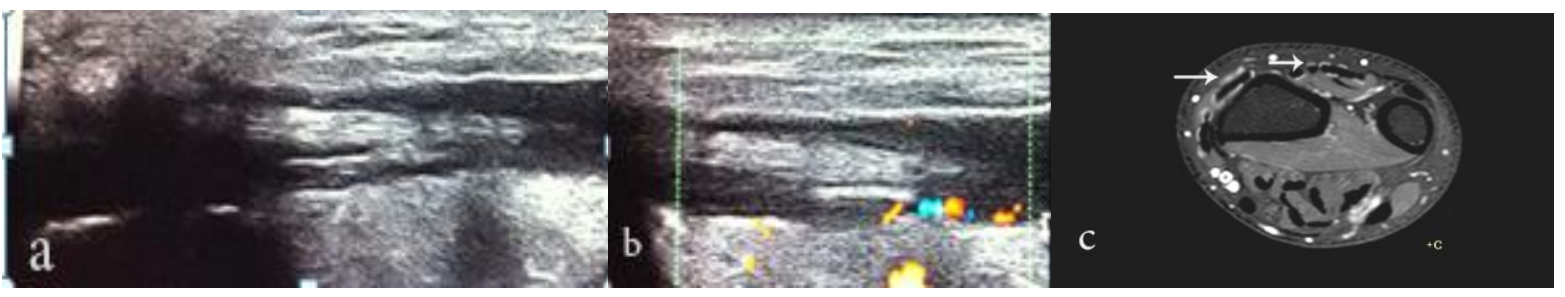

Figure 2. Imaging evidence of tenosynovitis. (a) Longitudinal ultrasonographic view of the extensor tendon at the level of the wrist reveals thickening of the synovial sheath. Color Doppler view demonstrates hyper vascularity of the synovial tissues (c) Contrast enhanced, axial, T1weighted, fat-saturated MR image of the distal forearm shows enhancement of the synovial sheath of the extensor tendons (arrows).

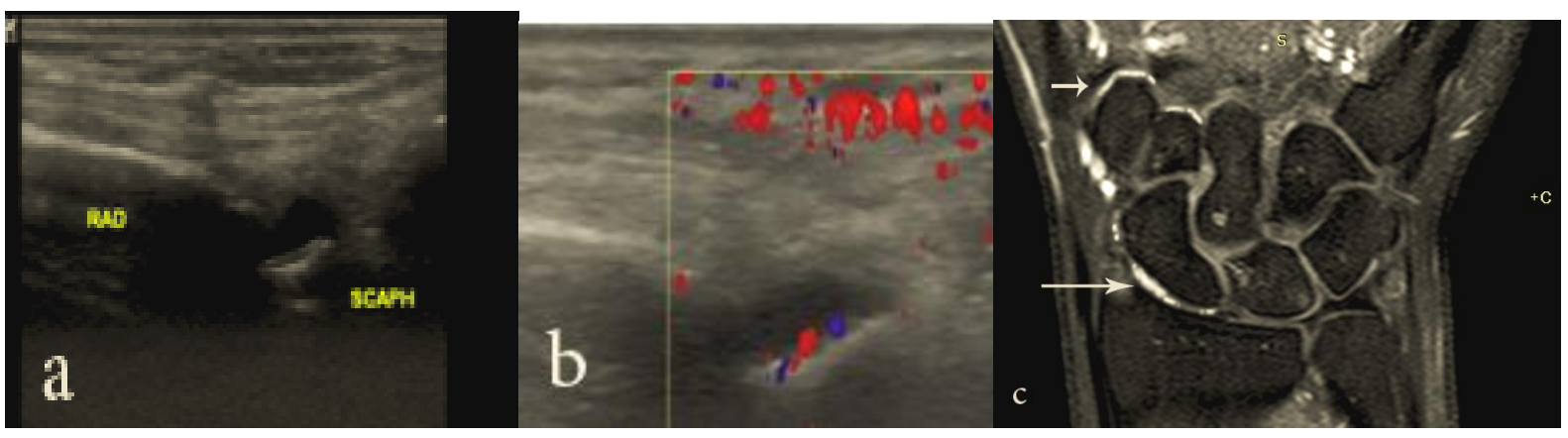

Figure 3. Imaging evidence of synovitis. (a) Longitudinal ultrasonographic view of the radio carpal joint shows synovial hypertrophy, with flow detected within the thickened synovium on color Doppler (b) (c) Contrast enhanced, Coronal, T1weighted, fat-saturated MR image of the distal forearm shows synovial enhancement of the left radiocarpal joint (long arrow), as well as synovial enhancement in some metacarpo-phalangeal joints (short arrow).
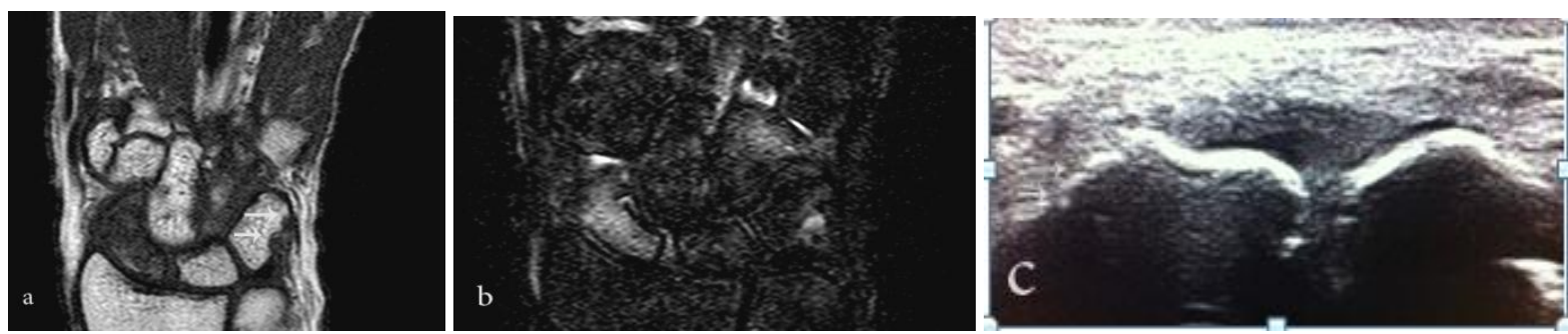

Figure 4. A Coronal T1WI image of the wrist showing low marrow signal involving the scaphoid and capitate bones, exhibiting bright signal on the coronal STIR images (b) consistent with bone marrow edema. Erosions of the triquetrum bone are also appreciated on the T1WI (arrows). (c) Sonographic image of the carpal bones, reveals the erosions seen in the triquetrum bone (arrows), yet bone marrow edema is not depicted on the ultrasound images. 
Table 4. Comparison between MSUS and MRI in detecting tenosynovitis, synovitis and erosions.

\begin{tabular}{lccc}
\hline & Tenosynovitis & Synovitis & Erosions \\
\hline MSUS & 11 & 10 & 7 \\
MRI & 16 & 15 & 11 \\
Percentage of agreement & 66 & 64 & 60 \\
\hline
\end{tabular}

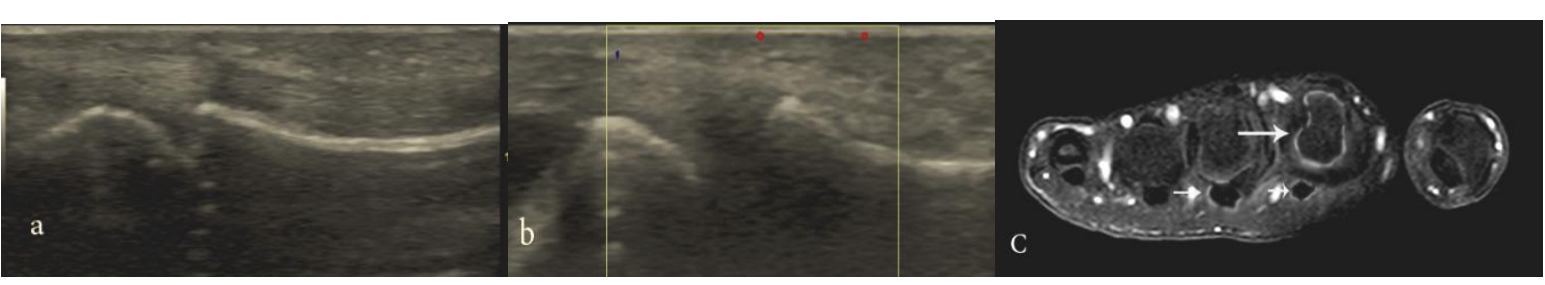

Figure 5. (a) Longitudinal ultrasonographic view of the palmar aspect of the second metacarpo-phalangeal joint showing a normal appearing joint, and overlying tendon with no evidence of synovitis or tenosynovitis. (b) No flow was detected within the joint or tendon sheath on color Doppler. (c) Contrast enhanced, axial, T1weighted, fat- saturated MR image of the hand at the level of metacarpo phalangeal joints shows minimal enhancement of the second metacarpo phalangeal joint (long arrow) and the synovial sheaths of the second and third finger flexor tendons (short arrows).

Table 5. Correlation between radiological findings and various disease parameters (only significant data shown).

\begin{tabular}{|c|c|c|c|}
\hline \multirow{2}{*}{ Variable } & \multicolumn{3}{|c|}{ Tenosynovitis / Synovitis } \\
\hline & $\mathbf{r}$ & $\mathbf{P}$ & $\mathbf{S}$ \\
\hline ESR & 0.44 & $<0.05$ & $\mathrm{~S}$ \\
\hline \multirow[t]{2}{*}{ CRP titer } & 0.53 & $<0.05$ & $S$ \\
\hline & \multicolumn{3}{|c|}{ Erosions } \\
\hline Disease duration & 0.63 & $<0.05$ & $\mathrm{~S}$ \\
\hline
\end{tabular}

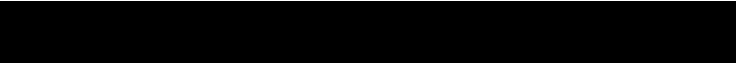

Joint symptoms have been noted in $12-66 \%$ of patients with systemic Sclerosis (SSc) at the time of diagnosis and in $24-97 \%$ of patients at some time during the course of their illness. Many radiological changes in the joints of SSc patients have been identified but erosive arthropathy is considered uncommon. ${ }^{8}$

Relatively few studies have examined the role of Musculoskeletal Ultrasonography (MSUS) and Magnetic Resonance Imaging (MRI) in the characterization of joint involvement in patients with SSc. ${ }^{9}$

In this study, MSUS and MRI were used to evaluate early inflammatory arthropathy in patients with SSc. The study included 20 patients with SSc, 4 males $(20 \%)$ and 16 females $(80 \%)$, mean age was 37.3 years (range 32-53 years) and mean disease duration was 8.7 years (range 6-19 years). All patients had arthralgia with no evidence of clinically swollen or tender joints. All patients were Rheumatoid factor (RF) and Anti CCP negative, ANA was positive in $95 \%$ of patients, CRP in $70 \%$ and the mean ESR was 37.3 \pm 24.16 .
In this study, MSUS detected tenosynovitis in 55\%, synovitis in $50 \%$ and erosions in $35 \%$ of patients as compared to MRI which detected tenosynovitis in $80 \%$, synovitis in $75 \%$ and erosions in $55 \%$ of the patients.

Recently, several surveys described a high frequency of musculoskeletal symptoms in patients with SSc. ${ }^{10,11}$ One study after assessing 45 consecutive patients with SSc for osteoarticular involvement using ultrasonography, stated that the prevalence of synovitis detected with ultrasound was significantly higher than that found by clinical examination. (26 vs 15 out of 45 cases, $\mathrm{P}=0.03$ ). ${ }^{12}$

In addition florid inflammatory changes with MRI have been reported in a significant proportion of symptomatic SSc patients, where inflammatory joint disease of the hand was assessed by MRI in 17 patients with a history of joint pain or swelling. Ten patients had inflammatory MRI findings with synovitis $(n=8)$, joint effusion $(n=7)$ or tenosynovitis $(n=8) .{ }^{13}$ However, their study cohort included patients with clinically swollen joints and positive serology for RF suggesting inclusion of patients with features of Rheumatoid arthritis overlap, which in contrast to this study, where at the time of 
assessment, the exclusion of patients with positive RF and anti CCP serology or clinically swollen joints allowed a reasonable assumption that our cohort did not include patients with features of overlap syndrome.

Relatively few studies however have compared between MSUS and MRI in the detection of inflammatory arthropathy in SSc patients. One study including $13 \mathrm{SSc}$ patients with arthralgia and no overt inflammatory arthritis had MSUS of their hands. 8 of these 13 patients also had MRI with gadolinium of their most symptomatic hand. Of the eight patients undergoing MRI scan, $100 \%$ of the patients had synovitis and $88 \%$ of patients had tenosynovitis. MRI also showed erosions in $75 \%$ of patients. With MSUS, tenosynovitis was seen in $47 \%$ of the patients and synovitis in $23 \%$, no erosions were identified. Comparison between MSUS and MRI showed 38\%, $62 \%$ and $25 \%$ percentage agreement for tenosynovitis, synovitis and erosions respectively. ${ }^{2}$

In agreement, a recent study of $16 \mathrm{SSc}$ patients compared MSUS and MRI in detecting synovial inflammation in SSc. With MSUS, tenosynovitis was detected in $7(43.7 \%)$ patients and synovitis in $5(31 \%)$ patients and erosions in 1 (6.3\%), Regarding MRI, 81.3 $\%$ (13) patients had tenosynovitis, $87.5 \%$ (14) patients had synovitis. and $62.5 \%$ (10) patients had erosions. Comparison between MSUS and MRI showed 62\%, $43 \%$ and $45 \%$ percentage agreement for tenosynovitis, synovitis and erosions respectively. The study concluded that while both MRI and MSUS are useful in characterizing synovial inflammation in SSc, MRI is clearly more sensitive than MSUS in this setting. ${ }^{4}$

In comparison with the previous two studies our results were somewhat similar as regard comparing MSUS and MRI for tenosynovitis and synovitis with a percentage agreement of $66 \%$ and $64 \%$ respectively. However our results for Erosions showed a higher percentage agreement $60 \%$, as compared to $25 \%$ and $45 \%$ in the previous two studies. The higher percentage of erosions detected by MSUS in this study could be explained by the wide availability of high resolution ultrasound equipment now available. In addition, bone erosions detected by MSUS and MRI in the present study showed a significant positive correlation with disease duration which could have influenced our findings.

In agreement with the present study, another study reported erosions in 53\% of patients with SSc studied by ultrasonography. ${ }^{14}$ Furthermore erosive arthropathy had been detected in 24 of 46 of SSc cases in a prior study. ${ }^{15}$

On the other hand however in two studies using MRI, hand erosions were detected in $16 \%(6 / 38)$ and $41 \%(7 / 17)$ of SSc patients. ${ }^{13}{ }^{16}$ Furthermore in one prospective cohort study involving 120 patients, erosive arthritis was found in only $22(18 \%)$ of SSc patients ${ }^{17}$ and in another longitudinal study, erosive arthritis of the hand was found in only one fourth of SSc patients. ${ }^{18}$
Review of the literature has revealed studies comparing sonography and MRI in erosive joint diseases. Alarcon and coworkers and Lopez-Ben and colleagues reported that ultrasonography had high accuracy, with MRI as the reference method, in the second and fifth MCP joints. ${ }^{19,20}$ In a group of patients with nonerosive RA on conventional radiography, Magnani and coworkers even visualized significantly more erosions in patients' MCP joints with ultrasonography than with MRI. ${ }^{21}$ Scheel et al found that ultrasound was superior to MRI in detecting erosions in the proximal interphalangeal joints but was less efficient at the metacarpo-phalangeal joints. ${ }^{22}$ However , It is important to mention that one of the earliest MRI signs of erosion is subchondral bone oedema, and there are, as yet, no studies to show that ultrasound is useful in detecting this. Ultrasound is not capable of appreciating intraosseous abnormalities that are not associated with an overlying surface defect. ${ }^{23}$

Several studies have correlated between the synovitis detected in SSc patients by MSUS and MRI, and acute phase reactants. In this study tenosynovitis and synovitis correlated significantly with ESR and CRP $(\mathrm{P}<0.05)$ and erosions correlated significantly with disease duration $(\mathrm{P}<0.05)$. These results were similar to a study that identified an association between synovitis and elevated ESR $(\geq 28 \mathrm{~mm} / \mathrm{h})$ and CRP $(\geq 10 \mathrm{mg} / \mathrm{l})$, suggesting that joint involvement might be associated with systemic inflammation in SSc. ${ }^{11}$ Furthermore in another multiple logistic regression analysis, higher ESR, age more than 38 and disease duration more than 10 years were risk factors for the occurrence of erosions. ${ }^{8}$

On the other hand however, other studies although reporting a high prevalence of joint inflammatory findings including synovitis, tenosynovitis and erosions in 17 symptomatic SSC patients undergoing MRI, yet they stated that the MRI findings did not correlate with any clinical or laboratory evidence of inflammation. ${ }^{13}$

In conclusion the current literature has shown that both ultrasound and MRI depicted musculoskeletal involvement in a considerable number of patients with systemic sclerosis, who showed no clinical evidence of inflammatory arthropathy. Advantages of MSUS over MRI include the ability to demonstrate angioneogenesis in active synovitis directly, whereas MRI requires injection of contrast medium. MSUS is a dynamic technique, with the ability to carry out rapid assessment of many widely spaced joints and to move and stress musculoskeletal structures. ${ }^{23}$ Unlike MRI, MSUS is readily suited to guiding intervention, such as small joint injection or synovial biopsy. There is also increasing evidence that patients prefer ultrasound to MRI. This may be related to claustrophobia but equally to the human interaction that occurs during an ultrasound examination, let alone the financial burden of a Post Contrast MRI study compared to a simple MSUS. ${ }^{24}$ 
Conversely, ultrasound is a relatively timeconsuming procedure, especially if multiple joints are examined. There is no ability to identify abnormalities overlooked and not imaged at the time of the original examination. Meanwhile, MRI has a higher sensitivity in detecting lesions, as well as a unique ability to display high resolution images, which can be obtained remotely, reviewed, re-read and scored at a later date and preserved for serial comparison.

Finally, given the burden of arthralgia and arthritis in SSc, randomized controlled trials are needed to evaluate associated joint involvement in SSc patients. In such trials, MSUS and MRI may be of major potential benefit in patient evaluation, where early detection and precise characterization of musculoskeletal involvement in such patients may be of good prognostic value.

\section{[Disclosure: Authors report no conflict of interest]}

\section{REFERENGES}

1. Stefan S, Jorg H, Anja U et al,: Magnetic resonance imaging findings in patients with systemic scleroderma and musculoskeletal symptoms. Eur Radiol 2013; 23:212-221.

2. Sarang C, Alessandro $\mathrm{C}$, Richard $\mathrm{H}$ et al,: Magnetic resonance imaging and musculoskeletal ultrasonography detect and characterize covert inflammatory arthropathy in systemic sclerosis patients with arthralagia. Rheumatology 2010; 49(12):2357-2361

3. Jerome A, Philip J, Dinesh K et al,: Articular involvement in systemic sclerosis. Rheumatology 2012; doi: 10.1093 kes041March 30.

4. Rasha A, Lotfi A and Ehab A,: Magnetic resonance imaging versus musculoskeletal ultrasonography in detecting inflammatory arthropathy in systemic sclerosis patients with hand arthralgia. Rheumatol Int 2013; 33(8):1961-1966.

5. Van den Hoogen F, Khanna D, Fransen $J$ et al,: 2013 classification criteria for systemic sclerosis; An American College of Rheumatology/European League against Rheumatism collaborative Initiative. Arthritis Rheum 2013; 65(11):2737-47.

6. Wakefield RJ, Balint PV, Szkudlarek M et al,: Musculoskeletal ultrasound including definitions for ultrasonographic pathology. J Rheumatol 2005; 32:2485-7

7. Wakefield RJ, Gibbon WW, Conaghan PG et al,: The value of sonography in the detection of bone erosions in patients with rheumatoid arthritis: a comparison with conventional radiography. Arthritis Rheum 2000; 43:2762-70.

8. Fadoua A, Latifa T, Adil $\mathrm{S}$ et al,: Erosive arthropathy in systemic sclerosis. BMC Public Health 2007; doi:10.1186/1471-2458-7-260

9. Chapin $\mathrm{R}$ and Hant FN: Imaging in scleroderma. Rheum Dis Clin North Am 2013; 39(3):515-46.
10. Bassel M, Hudson M, Taillefer SS et al,: Frequency and impact of symptoms experienced by patients with systemic sclerosis. Results from a Canadian national survey. Rheumatology (oxford) 2011; 50:62-767.

11. Avouac J, Walker U, Tyndall A et al,: Characteristics of joint involvement and relationships with systemic inflammation in systemic sclerosis. J Rhematol 2010; 37:1488-1501.

12. Cuomo G, Zappia M, Abignano $G$ et al,: Ultrasonographic features of the hand and wrist in Systemic Sclerosis. Rheumatology 2009; 48:1414-7

13. Low AH, Lax M, Johnson SR et al,: Magnetic resonance of the hand in systemic sclerosis. J Rheumatol 2009; 36:961-4.

14. Generini S, Steiner G, miniati I et al,: Anti RNP and other autoantibodies in syatemic sclerosis with joint involvement. Rheumatology (oxford) 2009; 48:920-25

15. La Montagna G, Sodano A, CapurroV et al,: The arthropathy of systemic sclerosis: a 12 month prospective clinical and imaging study. Skeletal Radiol 2005; 34:35-41

16. Allanore $\mathrm{Y}$, Seror R, Chevrot A et al,: Hand vascular involvement assessed by magnetic resonance angiography in systemic sclerosis. Arthritis Rheum 2007; 56:2747-54

17. Avouac J, Airo P, Dieude P et al,: Radiological hand involvement in systemic sclerosis. Ann Rheum dis 2006; 65:1088-92

18. Avouac J, Mogavero G, Guerini H et al,: Predictive factors of hand radiographic lesions in systemic sclerosis. Ann Rheum Dis 2011; 70:630-633.

19. Alarcon GS, Lopez-Ben R and Moreland LW: High-resolution ultrasound for the study of target joints in rheumatoid arthritis. Arthritis Rheum 2002; 46:1969-1970.

20. Lopez-Ben R, Bernreuter WK, Moreland LW et al,: Ultrasound detection of bone erosions in rheumatoid arthritis: a comparison to routine radiographs of the hands and feet. Skeletal Radiol 2004; 33:80-84.

21. Magnani $M$, Salizzoni E, Mule $\mathrm{R}$ et al,: Ultrasonography detection of early bone erosions in the metacarpophalageal joints of patients with rheumatoid arthritis. Clin Exp Rheumatol 2004; 22:743-748.

22. Scheel AK, Hermann KGA, Ohrndorf S, et al,: Prospective 7 year follow up imaging study comparing radiography, ultrasonography, and magnetic resonance imaging in rheumatoid arthritis finger joints. Ann Rheum Dis 2006; 65:595-600.

23. McNally EG: Ultrasound of the small joints of the hands and feet: current status. Skeletal Radiol 2008; 37:99-113

24. Middleton WD, Payne WT, Teefey SA et al,: Sonography and MRI of the shoulder: comparison of patient satisfaction. Am J Roentgenol 2004; 183 : $1449-1452$ 